\title{
Potential Therapeutic Drugs for Parkinson's Disease Based on Data Mining and Bioinformatics Analysis
}

\author{
Chuan Xu, ${ }^{1}$ Jiajun Chen, ${ }^{1}$ Xia Xu, ${ }^{2}$ Yingyu Zhang, ${ }^{1}$ and Jia Li ${ }^{1}{ }^{1}$ \\ ${ }^{1}$ Department of Neurology (III), China-Japan Union Hospital of Jilin University, Changchun 130033, Jilin, China \\ ${ }^{2}$ Yiwu Maternal and Child Health Hospital, Yiwu 322000, Zhejiang, China \\ Correspondence should be addressed to Jia Li; lijia33233@jlu.edu.cn
}

Received 16 May 2018; Revised 25 August 2018; Accepted 4 September 2018; Published 2 October 2018

Academic Editor: Jan Aasly

Copyright (c) 2018 Chuan Xu et al. This is an open access article distributed under the Creative Commons Attribution License, which permits unrestricted use, distribution, and reproduction in any medium, provided the original work is properly cited.

The objective is to search potential therapeutic drugs for Parkinson's disease based on data mining and bioinformatics analysis and providing new ideas for research studies on "new application of conventional drugs." Method differential gene candidates were obtained based on data mining of genes of PD brain tissue, original gene data analysis, differential gene crossover, pathway enrichment analysis, and protein interaction, and potential therapeutic drugs for Parkinson's disease were obtained through druggene relationship. Result. 250 common differential genes were obtained from 3 research studies, and 31 differential gene candidates were obtained through gene enrichment analysis and protein interaction. 10 drugs such as metformin hydrochloride were directly or indirectly correlated to differential gene candidates. Conclusion. Potential therapeutic drugs that may be used for prevention and treatment of Parkinson's disease were discovered through data mining and bioinformatics analysis, which provided new ideas for research and development of drugs. Results showed that metformin hydrochloride and other drugs had certain therapeutical effect on Parkinson's disease, and melbine (DMBG) can be used for treatment of Parkinson's disease and type 2 diabetes patients.

\section{Introduction}

With a high morbidity and a high disability rate, Parkinson's disease is the second degenerative disease of nervous system. Presently, treatment Parkinson's disease focuses on symptomatic treatment, which can just relief the symptoms and can neither effectively inhibit the progression of the disease nor cure it $[1,2]$. Research studies on "new application of conventional drugs" based on differential genes of the brain tissue may enable to cure Parkinson's disease (PD). Aspirin is a famous drug with "new application of conventional drugs." Aspirin was first applied to antipyretic-analgesic and anti-inflammatory treatment as a nonsteroidal antiinflammatory drug. Then, it was found to be able to inhibit antiplatelet aggregation of TXA2, and thus it has been extensively applied to treatment of cardiovascular and cerebrovascular diseases $[3,4]$. However, the mining model of indications for drug therapy was different from traditional drug R\&D modes. The latter depended on physical tests, such as cell test, animal test, and clinical test, which were made to determine chemical components of relevant substances, and synthesis of drug compounds and featured high investment, high risk, and long $\mathrm{R} \& \mathrm{D}$ cycle $[5,6]$. It was an alternative solution for drug $\mathrm{R} \& \mathrm{D}$ to readjust existing drugs for treatment of other diseases, guarantee of drug safety, lower cost, and higher R\&D efficiency [7].

This paper was aimed at providing new drug candidates for PD treatment and providing new methods and ideas for drug screening through data mining and bioinformatics analysis of PD brain tissue.

\section{Method}

(i) As shown in Figure 1, "PD (Parkinson's disease)" and "gene expression profiling" were retrieved in GEO dataset (gene expression omnibus dataset), and references were screened. Selection criteria for retrieved references: the approval of Ethics Committee was 


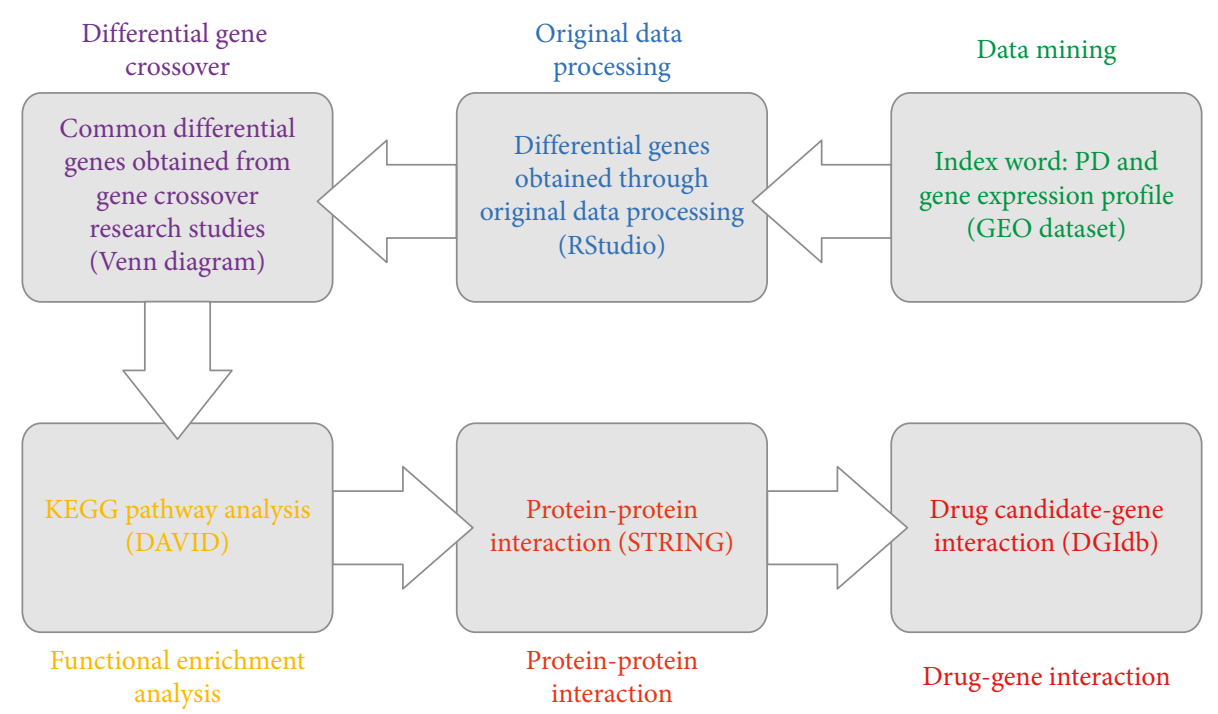

FIgure 1: Technical route of data mining.

indicated in the research; diagnosis of PD was demonstrated by clinic and neuropathology; the brain tissue of normal people and PD patients was the research object; original gene data can be obtained; original gene chip had high quality. Exclusion criteria: the approval of Ethics Committee was not indicated in the research; diagnosis of PD was not demonstrated by clinic and neuropathology; the research object was not the brain tissue of normal people and PD patients; original gene data cannot be obtained; original gene chip had poor quality. Screened original gene data was obtained and downloaded.

(ii) The quality of original gene data was evaluated using $\mathrm{R}$ language and RStudio software, and $\log \mathrm{FC}>1$ or $\log \mathrm{FC}<(-1)$ was set to obtain differential genes.

(iii) Venn diagram of differential genes obtained in the research studies using bioinformatics and evolutionary genomics (http://bioinformatics.psb.ugent. be/webtools/venn/), and common differential genes were obtained.

(iv) KEGG pathway analysis of common differential genes was made using DAVID (https://david. ncifcrf.gov/) and differential genes closely related to $\mathrm{PD}$ were screened [8].

(v) Protein-protein interaction of genes closely related to PD was figured out using STRING (https://string$\mathrm{db} . \mathrm{org} /$ ), so as to make the protein-protein interaction closer and reduce the range of differential gene candidates. Confidence level $\geq 0.90$ was set using STRING and protein-protein interactions, and differential gene candidates were obtained [9].

(vi) Differential gene candidates were inputted into DGIdb dataset (http://dgidb.genome.wustl.edu/) so as to obtain the interrelation between drug and gene and drug candidates for treatment of PD. Relevant information of drug was obtained using PubChem dataset, the approval for clinic or clinical test was searched in ClinicalTrials dataset, and drug candidates were analyzed $[10,11]$.

\section{Result}

One hundred sixty-three retrieved results were obtained from the retrieval of "PD (Parkinson's disease)" and "gene expression profiling" in GEO dataset, and based on strict screening, 3 of them conformed the screening requirements and can be applied to our research studies (Moran et al. [12] (chip number: GSE8397); Lewandowski et al. [13] (chip number: GSE19587); Edna et al. [14] (chip number: GSE20333)). 3 searches were analyzed using RStudio software, and 1191, 4484, and 2173 differential genes were obtained, respectively. The Venn diagram of 3 groups of differential genes was drawn using "bioinformatics and evolutionary genomics," as shown in Figure 2, and 250 common differential genes were obtained.

KEGG pathway analysis was made for 250 differential genes using DAVID, and pathways whose $P$ value was less than 0.05 were screened. Figure 3 showed KEGG pathway analysis of 250 differential genes whose $P$ value was less than 0.05. Metabolic pathways, carbon metabolisms, cysteine and methionine metabolism, and Parkinson's disease were 4 KEGG pathways with a smaller $P$ value, and they contained $51,12,7$, and 12 differential genes, respectively, as shown in Table 1. 82 differential genes of the aforesaid 4 pathways were further analyzed.

Protein-protein interaction of 82 differential genes of the aforesaid 4 pathways was obtained using STRING, and confidence level $\geq 0.90$ was set in STRING so as to ensure proteins were closely related. As shown in Figure 4, 31 proteins were closely connected. It indicated that the drug had direct or indirect effect on 31 proteins when it acted on one or more proteins.

31 differential gene candidates were inputted into DGIdb dataset so as to obtain the drug interacting with the aforesaid genes, and 10 drug candidates were obtained through 


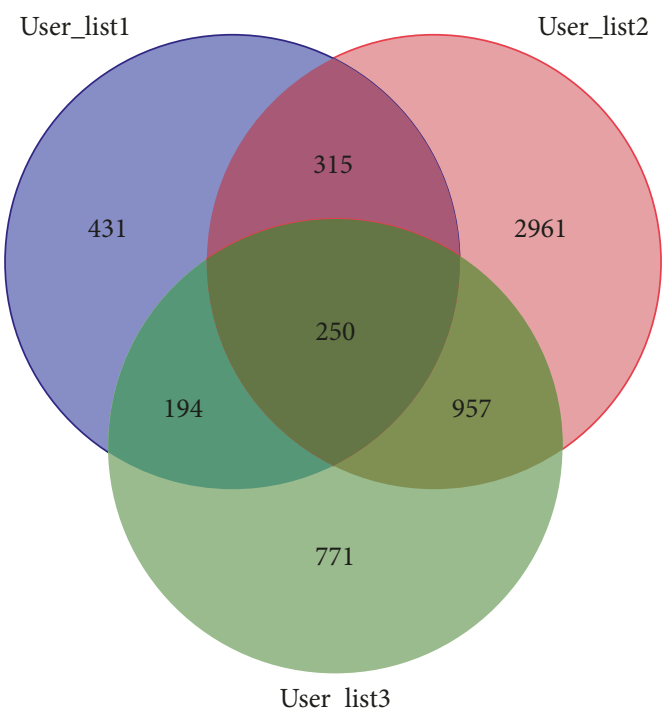

Figure 2: Venn diagram of 3 groups of differential genes. List 1, list 2, and list 3 were differential genes of chips GSE8397, GSE19587 and GSE8397, and GSE20333 respectively. 431, 2961, and 771 genes of 3 groups were identified by bioinformatics and evolutionary genomics, respectively, and 3 groups had 250 common differential genes.

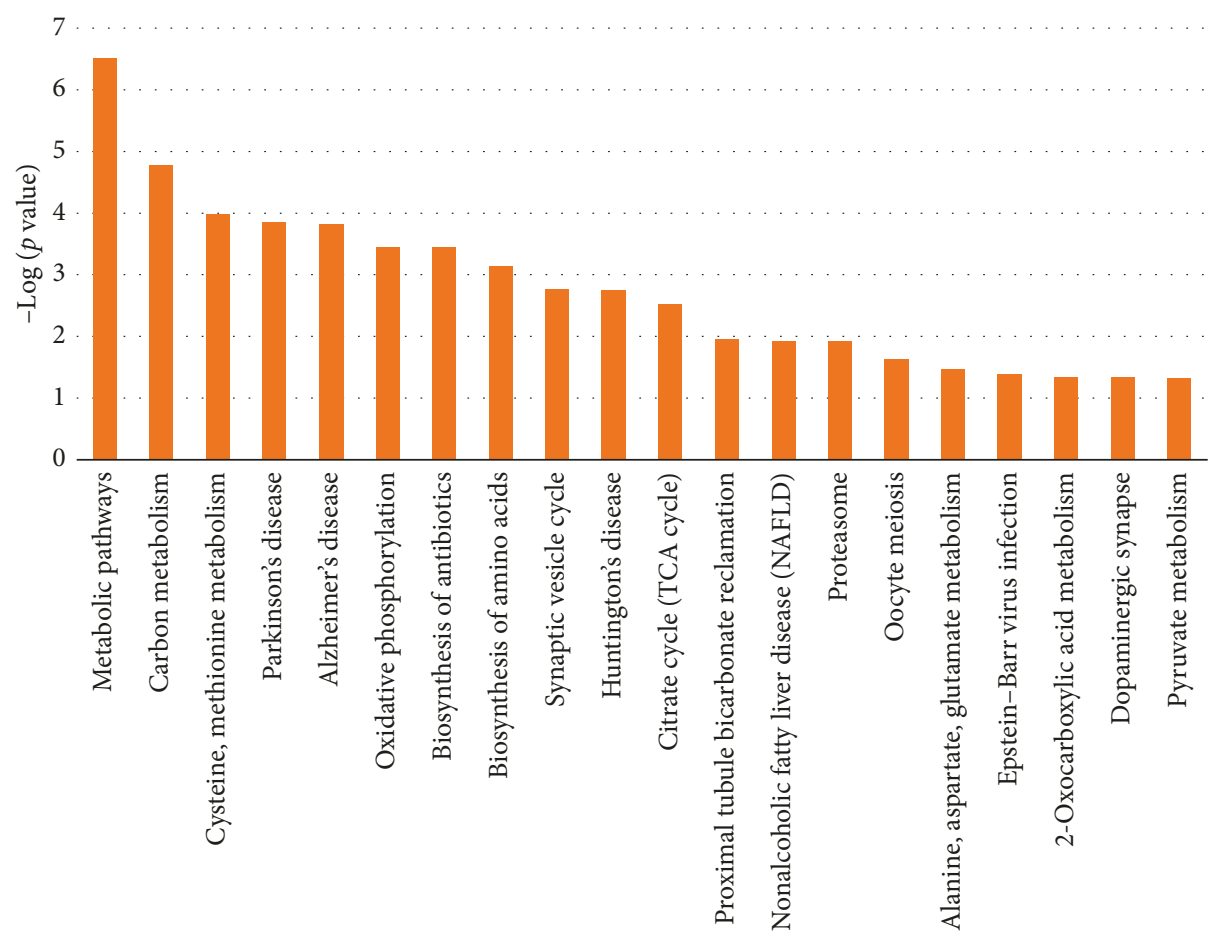

KEGG pathway analysis

Figure 3: KEGG pathway analysis of 250 differential genes whose $P$ value was less than 0.05 .

screening their biological characteristics and clinical application. As shown in Table 2, 10 drugs, such as metformin hydrochloride, were directly or indirectly correlated to differential gene candidates.

\section{Conclusions}

Drugs that may be used for prevention and treatment of PD were discovered through data mining and pathway analysis, which provided new ideas for drug R\&D.
Based on the research result and information retrieval relating to drug candidates, 10 drugs such as metformin hydrochloride had certain therapeutical effect on PD, and melbine (DMBG) can be used for treatment of PD and type 2 diabetes patients.

\section{Discussion}

PD had a high morbidity, and drug therapy represented by dopaminergic drugs was a main treatment method for PD 
Table 1: Differential genes of 4 DAVID KEGG pathways with a smaller $P$ value.

\begin{tabular}{|c|c|c|c|}
\hline KEGG pathway & Gene number & $P$ value & Genes \\
\hline Metabolic pathways & 51 & $3.08 E-07$ & $\begin{array}{l}\text { UQCRC2, SGSH, LDHA, IMPA1, HMGCR, ATP5B, } \\
\text { CYC1, GSS, ALAS1, IDH3G, GOT1, PIGB, PTDSS1, } \\
\text { NDUFS1, DHCR24, PLD3, CMAS, PFKP, PFKM, } \\
\text { DGUOK, CDO1, NDUFA10, SDS, OAT, MDH2, } \\
\text { MDH1, ME3, NDUFB5, SORD, GLUD1, UROS, } \\
\text { ASNS, ATP6V1B2, ALDH1A1, ENO2, PAFAH1B1, } \\
\text { PTS, PDHX, MTMR4, POLR3F, MSMO1, MOCS2, } \\
\text { NDUFA9, NDUFA7, IDH3B, ACLY, ATP6V1E1, } \\
\text { NDUFV2, MTR, QPRT, HIBCH }\end{array}$ \\
\hline Carbon metabolism & 12 & $1.70 E-0.5$ & $\begin{array}{l}\text { ME3, GOT1, IDH3G, SDS, GLUD1, ENO2, PFKP, } \\
\text { IDH3B, HIBCH, PFKM, MDH2, MDH1 }\end{array}$ \\
\hline Cysteine and methionine metabolism & 7 & $1.05 E-0.4$ & $\begin{array}{l}\text { LDHA, GOT1, SDS, MTR, CDO1, MDH2, MDH1 } \\
\text { UQCRC2, NDUFB5, NDUFA9, ATP5B, CYC1, }\end{array}$ \\
\hline Parkinson's disease & 12 & $1.40 E-0.4$ & $\begin{array}{c}\text { NDUFA7, NDUFV2, UCHL1, SLC18A2, NDUFA10, } \\
\text { VDAC3, NDUFS1 }\end{array}$ \\
\hline
\end{tabular}

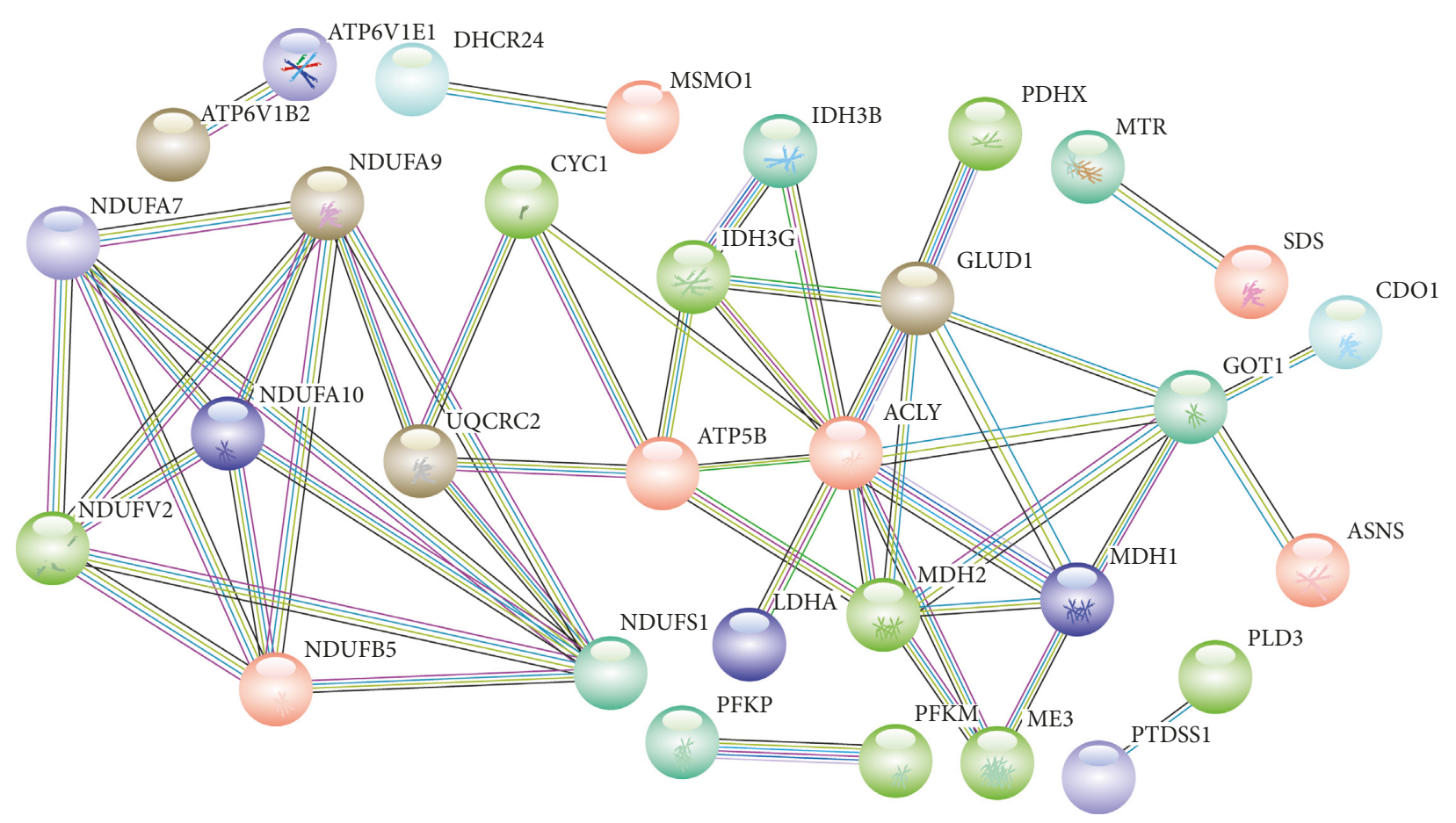

FIgURE 4: Thirty-one protein-protein correlations.

[15]. Nevertheless, anti-Parkinson's disease drugs mainly acted on dopamine metabolism and cholinergic metabolic pathways instead of the apoptosis mechanism of dopaminergic neuron, so it can neither inhibit the progress of the disease effectively nor cure the disease. Whereas traditional drugs synthesized based on physical tests and drug compounds featured long R\&D cycle, high investment, high risk, and poor curative effect of clinical test, anti-Parkinson's disease drugs fell behind in drug R\&D and could not accommodate to the rapid growth of PDs [16].

Data mining was an emerging research area in recent years that aimed to excavate potential and possible data pattern, internal relation, rule and development trend, etc. from unorganized data information, extract effective, novel, useful, understandable, and scattered valuable knowledge from text files and to make use of such knowledge for better information organization. Data mining of biomedical literature was construed as effective formation of research hypothesis, since it can reveal a new relationship between gene and pathogenesis. New evidences of adjustment of existing indications for drug therapy can be obtained through data mining combined with other bioinformatics tools [17-19] and reliable conclusions were drawn from drug R\&D based on data mining.

In this research, macromining and microanalysis were combined innovatively, the direction of drug screening was 
TABLE 2: Thirty-one drug candidates for differential gene prediction (drug-gene connection table).

\begin{tabular}{|c|c|c|c|c|c|c|}
\hline Drug & Gene & $\begin{array}{l}\text { Interaction } \\
\text { types }\end{array}$ & Approved? & Administration & Approved use & PubMed ID \\
\hline Citric acid & $\mathrm{MDH} 2$ & N/A & Yes & Oral administration & Anticoagulation & 10592235 \\
\hline Folic acid & MTR & N/A & yes & Oral administration & Trophic nerve & \\
\hline Hydroxocobalamin & MTR & Cofactor & Yes & $\begin{array}{c}\text { Oral } \\
\text { administration/intravenous } \\
\text { drip }\end{array}$ & Neuroprotection & $\begin{array}{c}18565,1744096 \\
7599160,3812589 \\
9587031\end{array}$ \\
\hline L-glutamate & $\begin{array}{c}\text { ASNS, GLUD1, } \\
\text { GOT1 }\end{array}$ & N/A & Phase 3 & Oral administration & Neuroprotection & $\begin{array}{c}17139284, \\
17016423,8288265, \\
17139284, \\
17016423, \\
17444813\end{array}$ \\
\hline $\begin{array}{l}\text { Metformin } \\
\text { hydrochloride }\end{array}$ & $\begin{array}{l}\text { NDUFA10, } \\
\text { NDUFA7, } \\
\text { NDUFA9, } \\
\text { NDUFB5, } \\
\text { NDUFS1, } \\
\text { NDUFV2 }\end{array}$ & Inhibitor & Yes & Oral administration & $\begin{array}{c}\text { Hypoglycemic } \\
\text { effect }\end{array}$ & \\
\hline Methionine & MTR & Product & Yes & Oral administration & Liver protection & $\begin{array}{l}17222188, \\
16618098, \\
17615995, \\
16788729, \\
17052662\end{array}$ \\
\hline Niacinamide & LDHA & N/A & Yes & Oral administration & $\begin{array}{l}\text { Cardiac disease, } \\
\text { cognitive } \\
\text { disorder }\end{array}$ & $\begin{array}{l}10592235 \\
17139284 \\
17016423\end{array}$ \\
\hline $\begin{array}{l}\text { Pyridoxal } \\
\text { phosphate }\end{array}$ & GOT1, SDS & Activator & Phase 2 & Oral administration & Dyskinesia & $\begin{array}{l}11340119, \\
16925884, \\
12167474, \\
11888303, \\
11752352, \\
14596599, \\
15155761, \\
14646100, \\
16580895, \\
15689518\end{array}$ \\
\hline Quercetin & ATP5B & N/A & Phase 1 & Oral administration & Pain & 10592235 \\
\hline Serine & SDS & N/A & Phase 2 & Oral administration & $\begin{array}{c}\text { Cognitive } \\
\text { improvement }\end{array}$ & $\begin{array}{c}4377655,14688104, \\
17139284, \\
17016423,500557\end{array}$ \\
\hline
\end{tabular}

determined, and targeting and high efficiency of drug mining were guaranteed based on big data analysis, bioinformatics analysis, and molecular pathology. 3 research studies were included in this research based on data mining of PD in GEO dataset. 250 differential genes were obtained from 3 groups of differential genes after gene crossover based on data mining of original gene and underwent KEGG analysis. KEGG pathway included Parkinson's disease and other pathways. 82 differential genes selected from 4 KEGG pathways with a smaller $P$ value were further analyzed in order to ensure the reliability of differential gene candidates, drug-gene correlation of 31 differential gene candidates was analyzed, and finally 10 drug candidates were screened out.

Some potential therapeutic drugs for PD were discovered in our research studies, among which melbine (DMBG) was worth noting. Current research studies showed that mitochondrial function disorder, abnormal protein aggregation, neuroinflammation increase, and impaired cerebral glucose metabolism were common processes of insulin resistance, diabetes, and nervous system degeneration and have been identified as the key mechanism for the progress of PD and cognitive disorder [20]. Besides, it has been considered that melbine (DMBG) cannot be applied to treatment of type 2 diabetes through adjustment of glucose metabolism disorder, but it had obvious protective effect on the nerve cells of $\mathrm{PD}$ and other nervous system degenerations [21]. This research showed that metformin hydrochloride can be combined with NDUFA10, NDUFA7, NDUFA9, NDUFB5, NDUFS1, NDUFV2, and other acceptors and thus affect the mitochondrial respiratory chain. Information retrieval indicated that melbine (DMBG) had therapeutic effect on the animal model of PD, and epidemiological survey also indicated that it had effect on prevention and treatment of PD. Mark et al. carried out epidemiological survey on type 2 diabetes patients with PD and found that the probability of type 2 diabetes patients suffering PD was 2.2 times higher than normal people. However, melbine (DMBG) could control blood glucose so as to reduce the probability of type 2 
diabetes patients suffering PD [22, 23]. Kang et al. discovered that melbine (DMBG) could mediate ATF2/CREB-PGC- $1 \alpha$ pathway, induce proteomic change of metabolisms and mitochondria pathways in the substantia nigra, increase mitochondrial protein in the substantia nigra and the corpus striatum, protect dopaminergic neuron in the substantia nigra and the corpus striatum, and improve dyskinesia of PD [24]. Julia et al. discovered that TRAP1 could adjust the mitochondrial function of downstream PINK1 and HTRA2, malfunction of TRAP1 increased free NADH, mitochondria was produced, unfolded protein reaction and membrane potential of mitochondria were triggered, the sensitivity of mitochondria elimination and apoptosis decreased, and PD patients suffered TRAP1 malfunction, while metformin hydrochloride could adjust energy metabolism, produce mitochondria, recover the mitochondrial membrane potential, reverse mitochondrial mitochondrial function arising from TRAP1 mutation of PD, and provide new ideas for mitochondrial pathological change and treatment of PD $[22,25,26]$. Nevertheless, metformin hydrochloride had many untoward effects. Gastrointestinal reaction was the most common untoward effect, and other uncommon untoward effects included lactic acidosis, cutaneous anaphylaxis, hepatorenal damage, hypoglycemia, hematological damage, acute pancreatitis, neural abnormity, etc. [27, 28]. Therefore, metformin hydrochloride may have great clinical effect on treatment of $\mathrm{PD}$, but we suggested using melbine (DMBG) for treatment of PD with type 2 diabetes and paying attention to the side effects.

Other than melbine (DMBG), other potential therapeutic drugs for PD were also discovered in this research. Research studies showed that PD patients lacked V12 but had higher HCY (homocysteine), so V12 could improve cognitive disorder and other symptoms of PD [29]. Clinical tests on vitamin B12 for treating cognitive disorder and other nonmotor symptoms of PD have been terminated [30]. In this research, hydroxocobalamin (namely vitamin B12) had mutual effect with MTR and affected transmethylation of methyl cobalamin to HCY (homocysteine) and consequently it gave play to neuroprotection. Folic acid was similar to hydroxocobalamin, both were the substrates of nerve regression and showed the potential to improve nonmotion functional disorder of PD. Methionine took part in many important physiological processes of human body, it has been rarely reported on PD and may have the potential to treat PD. Citric acid was an anticoagulant drug that took part in tricarboxylic acid cycle, affected the production of mitochondrial energy, and had a certain potential to treat PD [31]. L-glutamate interacted with ASNS, GLUD1, and GOT1. The interaction between dopamine and glutamic acid in the basal ganglion played an important part in the adjustment of motion and cognitive behavior, and functional disorder of dopaminergic-glutamic acid pathway was discovered in PD pathology [32-34]. Serine was an amino acid and research studies indicated that amino acid of D-serine could improve behavior and motor symptoms of PD $[35,36]$. Moreover, niacinamide interacted with LDHA. Research studies showed that niacinamide protected dopaminergic neuron from neural degeneration induced by
MPTP in the PD rat model, and it had protective effect on dopaminergic neuron [37, 38]. Niacinamide was a constituent part of coenzyme I and coenzyme II and became a coenzyme of many dehydrogenase. It has been approved to be used for prevention of endemic erythema and aniacinosis. Meanwhile, niacinamide could improve cognitive disorder of Alzheimer disease and has entered the second clinical test stage [39]. Pyridoxal phosphate, namely, vitamin B6, was a coenzyme of aminopherase and decarboxylase in the amino acid metabolism that could promote decarboxylation of glutamic acid. It has been discovered that oral administration of a moderate amount of vitamin B6 had preventive effect on PD [40, 41]. Furthermore, pyridoxal phosphate could relieve tardive dyskinesia of schizophrenia and schizoaffective disorder patients and has entered the second test stage [42]. Quercetin was an active component of many traditional Chinese medicines. It has been approved to be used for reducing the friability of blood corpuscle and had neuroprotective effect on dopaminergic neuron due to antioxygenation $[43,44]$.

This research still has some disadvantages. It is currently believed that Parkinson's disease is a neurodegenerative disease, caused by a combination of genetic factors, environmental factors, and nervous system aging, through oxidative stress, proteasome dysfunction, inflammation/immune response, mitochondrial dysfunction, calcium homeostasis, excitatory toxins, apoptosis, etc., leading to the degeneration and loss of substantia nigra dopaminergic neurons [45]. The susceptibility gene of Parkinson's disease is part of the pathogenic factor, so the efficacy of Parkinson's disease-based therapeutic drugs based on the Parkinson's disease susceptibility gene needs to be further evaluated. 3 research studies failed to identify the drugs for PD during the lifetime, so differential genes between PD group and the control group may be caused by other pathogenic factors. The drug candidates failed to further verify the potential therapeutical effect on PD. However, this research provided new ideas for R\&D of PD drugs and potentially safe drugs for treatment of PD.

In short, drugs that may be used for prevention and treatment of PD can be obtained from data mining and bioinformatics analysis, which provided new ideas for drug $\mathrm{R} \& \mathrm{D}$ and research studies on "new application of conventional drugs." Metformin hydrochloride and other drugs had great clinical effect on treatment of PD, so melbine (DMBG) can be used for treatment of PD and type 2 diabetes patients, which can be proved by more clinical tests.

\section{Data Availability}

All data reported in this manuscript are included within the article. Raw data, if required, are available on request.

\section{Conflicts of Interest}

The authors declare that they have no conflicts of interest.

\section{References}

[1] T. Capriotti and K. Terzakis, "Parkinson disease," Home Healthcare Now, vol. 34, no. 6, pp. 300-307, 2016. 
[2] J. M. Ellis and M. J. Fell, "Current approaches to the treatment of Parkinson's disease," Bioorganic \& Medicinal Chemistry Letters, vol. 27, no. 18, pp. 4247-4255, 2017.

[3] J. Y. Wick, "Aspirin: a history, a love story," Consultant Pharmacist, vol. 27, no. 5, pp. 322-329, 2012.

[4] M. J. R. Desborough and D. M. Keeling, "The aspirin storyfrom willow to wonder drug," British Journal of Haematology, vol. 177, no. 5, pp. 674-683, 2017.

[5] A. Ranga, N. Gjorevski, and M. P. Lutolf, "Drug discovery through stem cell-based organoid models," Advanced Drug Delivery Reviews, vol. 69-70, pp. 19-28, 2014.

[6] A. Astashkina and D. W. Grainger, "Critical analysis of 3-D organoid in vitro cell culture models for high-throughput drug candidate toxicity assessments," Advanced Drug Delivery Reviews, vol. 69-70, pp. 1-18, 2014.

[7] J. Kirk, N. Shah, B. Noll et al., "Text mining-based in silico drug discovery in oral mucositis caused by high-dose cancer therapy," Supportive Care in Cancer, vol. 26, no. 8, pp. 2695-2705, 2018.

[8] D. W. Huang, B. T. Sherman, Q. Tan et al., "DAVID bioinformatics resources: expanded annotation database and novel algorithms to better extract biology from large gene lists," Nucleic Acids Research, vol. 35, no. 2, pp. W169-W175, 2007.

[9] D. Szklarczyk, A. Franceschini, S. Wyder et al., "STRING v10: protein-protein interaction networks, integrated over the tree of life," Nucleic Acids Research, vol. 43, pp. D447-D452, 2015.

[10] K. C. Cotto, A. H. Wagner, Y.-Y. Feng et al., "DGIdb 3.0: a redesign and expansion of the drug-gene interaction database," Nucleic Acids Research, vol. 46, no. D1, pp. D1068D1073, 2017.

[11] G. Fu, C. Batchelor, M. Dumontier, J. Hastings, E. Willighagena, and E. Bolton, "PubChemRDF: towards the semantic annotation of PubChem compound and substance databases," Journal of Cheminformatics, vol. 7, no. 1, p. 34, 2015.

[12] L. B. Moran, D. C. Duke, M. Deprez, D. T. Dexter, R. K. Pearce, and M. B. Graeber, "Whole genome expression profiling of the medial and lateral substantia nigra in Parkinson's disease," Neurogenetics, vol. 7, no. 1, pp. 1-11, 2006.

[13] N. M. Lewandowski, S. Ju, M. Verbitsky et al., "Polyamine pathway contributes to the pathogenesis of Parkinson disease," Proceedings of the National Academy of Sciences, vol. 107, no. 39, pp. 16970-16975, 2010.

[14] G. Edna, M. Silvia, J. Jasmine et al., "Gene expression profiling of parkinsonian substantia nigra," GEO, 2010.

[15] A. Affini, S. Hagenow, A. Zivkovic, J. Marco-Contelles, and H. Stark, "Novel indanone derivatives as MAO B/H3R dualtargeting ligands for treatment of Parkinson's disease," European Journal of Medicinal Chemistry, vol. 148, pp. 487-497, 2018.

[16] B. Thrash, K. Thiruchelvan, M. Ahuja, V. Suppiramaniam, and M. Dhanasekaran, "Methamphetamine-induced neurotoxicity: the road to Parkinson's disease," Pharmacological Reports, vol. 61, no. 6, pp. 966-977, 2009.

[17] E. L. Berg, "Systems biology in drug discovery and development," Drug Discovery Today, vol. 19, no. 2, pp. 113-125, 2014.

[18] E. Mosca, G. Bertoli, E. Piscitelli et al., "Identification of functionally related genes using data mining and data integration: a breast cancer case study," BMC Bioinformatics, vol. 10, no. 12, p. S8, 2009.
[19] S. Yu, L.-C. Tranchevent, B. De Moor, and Y. Moreau, "Gene prioritization and clustering by multi-view text mining," $B M C$ Bioinformatics, vol. 11, no. 1, p. 28, 2010.

[20] M. R. Ashraghi, G. Pagano, S. Polychronis, F. Niccolini, and M. Politis, "Parkinson's disease, diabetes and cognitive impairment," Recent Patents on Endocrine, Metabolic \& Immune Drug Discovery, vol. 10, no. 1, pp. 11-21, 2016.

[21] B. Viollet, B. Guigas, N. S. Garcia, J. Leclerc, M. Foretz, and F. Andreelli, "Cellular and molecular mechanisms of metformin: an overview," Clinical Science, vol. 122, no. 6, pp. $253-270,2012$.

[22] M. L. Wahlqvist, M.-S. Lee, C.-C. Hsu, S.-Y. Chuang, J.-T. Lee, and H.-N. Tsai, "Metformin-inclusive sulfonylurea therapy reduces the risk of Parkinson's disease occurring with type 2 diabetes in a Taiwanese population cohort," Parkinsonism \& Related Disorders, vol. 18, no. 6, pp. 753-758, 2012.

[23] M. L. Wahlqvist, M.-S. Lee, S.-Y. Chuang et al., "Increased risk of affective disorders in type 2 diabetes is minimized by sulfonylurea and metformin combination: a population-based cohort study,” BMC Medicine, vol. 10, no. 1, p. 150, 2012.

[24] H. Kang, R. Khang, S. Ham et al., "Activation of the ATF2/ CREB-PGC-1alpha pathway by metformin leads to dopaminergic neuroprotection," Oncotarget, vol. 8, no. 30, pp. 48603-48618, 2017.

[25] J. C. Fitzgerald, A. Zimprich, D. A. C. Berrio et al., "Metformin reverses TRAP1 mutation-associated alterations in mitochondrial function in Parkinson's disease," Brain, vol. 140, no. 9, pp. 2444-2459, 2017.

[26] H. Kim, J. Yang, M. J. Kim et al., "Tumor necrosis factor receptor-associated protein 1 (TRAP1) mutation and TRAP1 inhibitor gamitrinib-triphenylphosphonium (G-TPP) induce a forkhead box o (FOXO)-dependent cell protective signal from mitochondria," Journal of Biological Chemistry, vol. 291, no. 4, pp. 1841-1853, 2016.

[27] S. Alusik and Z. Paluch, "Metformin: the past, presence, and future," Minerva Medica, vol. 106, no. 4, pp. 233-238, 2015.

[28] The American College of Physicians, "Summaries for patients: oral drug treatment of type 2 diabetes mellitus: a clinical practice guideline from the American College of Physicians," Annals of Internal Medicine, vol. 156, no. 3, p. I36, 2012.

[29] Y. Xie, H. Feng, S. Peng, J. Xiao, and J. Zhang, "Association of plasma homocysteine, vitamin B12 and folate levels with cognitive function in Parkinson's disease: a meta-analysis," Neuroscience Letters, vol. 636, pp. 190-195, 2017.

[30] F. Cardoso, "Vitamin B12 and Parkinson's disease: what is the relationship?," Movement Disorders, vol. 33, no. 5, pp. 702-703, 2018.

[31] L. P. Leow, L. Beckert, T. Anderson, and M.-L. Huckabee, "Changes in chemosensitivity and mechanosensitivity in aging and Parkinson's disease," Dysphagia, vol. 27, no. 1, pp. 106-114, 2012.

[32] M. Amalric, "Targeting metabotropic glutamate receptors (mGluRs) in Parkinson's disease," Current Opinion in Pharmacology, vol. 20, pp. 29-34, 2015.

[33] I. Obal et al., "Mental disturbances in Parkinson's disease and related disorders: the role of excitotoxins," Journal of Parkinson's Disease, vol. 4, no. 2, pp. 139-150, 2014.

[34] S. D. Pizzi, R. G. Bellomo, S. M. Carmignano et al., "Rehabilitation program based on sensorimotor recovery improves the static and dynamic balance and modifies the basal ganglia neurochemistry: a pilot $1 \mathrm{H}-\mathrm{MRS}$ study on Parkinson's disease patients," Medicine, vol. 96, no. 50, article e8732, 2017.

[35] E. Gelfin, Y. Kaufman, I. Korn-Lubetzki et al., "D-serine adjuvant treatment alleviates behavioural and motor 
symptoms in Parkinson's disease," International Journal of Neuropsychopharmacology, vol. 15, no. 4, pp. 543-549, 2012.

[36] T. Clairembault, W. Kamphuis, L. Leclair-Visonneau et al., "Enteric GFAP expression and phosphorylation in Parkinson's disease," Journal of Neurochemistry, vol. 130, no. 6, pp. 805-815, 2014.

[37] J. Xu, SQ. Xu, J. Liang, Y. Lu, J. H. Luo, and J. H. Jin, "Protective effect of nicotinamide in a mouse Parkinson's disease model," Journal of Zhejiang University, vol. 41, no. 2, pp. 146-152, 2012.

[38] S. Lehmann, A. C. Costa, I. Celardo, S. H. Y. Loh, and L. M. Martins, "Parp mutations protect against mitochondrial dysfunction and neurodegeneration in a PARKIN model of Parkinson's disease," Cell Death \& Disease, vol. 7, no. 3, article e2166, 2016.

[39] C. Allone, V. Lo Buono, F. Corallo et al., "Cognitive impairment in Parkinson's disease, Alzheimer's dementia, and vascular dementia: the role of the clock-drawing test," Psychogeriatrics, vol. 18, no. 2, pp. 123-131, 2018.

[40] L. Shen, "Associations between B vitamins and Parkinson's disease," Nutrients, vol. 7, no. 9, pp. 7197-7208, 2015.

[41] Y. Sato, C. Yasumiishi, T. Chiba, and K. Umegaki, "A systematic review to identify unacceptable intake levels of vitamin B6 among patients taking levodopa," Food Hygiene and Safety Science, vol. 58, no. 6, pp. 268-274, 2017.

[42] A. O. Adelufosi, O. Abayomi, and T. M. Ojo, "Pyridoxal 5 phosphate for neuroleptic-induced tardive dyskinesia," Cochrane Database of Systematic Reviews, vol. 4, article CD010501, 2015.

[43] K. M. D. Joseph and Muralidhara, "Combined oral supplementation of fish oil and quercetin enhances neuroprotection in a chronic rotenone rat model: relevance to Parkinson's disease," Neurochemical Research, vol. 40, no. 5, pp. 894-905, 2015.

[44] M. Ay, J. Luo, M. Langley et al., "Molecular mechanisms underlying protective effects of quercetin against mitochondrial dysfunction and progressive dopaminergic neurodegeneration in cell culture and MitoPark transgenic mouse models of Parkinson's Disease," Journal of Neurochemistry, vol. 141 , no. 5, pp. 766-782, 2017.

[45] A. J. Lees, J. Hardy, and T. Revesz, "Parkinson's disease," The Lancet, vol. 373, no. 9680, pp. 2055-2066, 2009. 


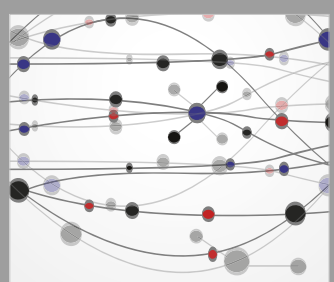

The Scientific World Journal
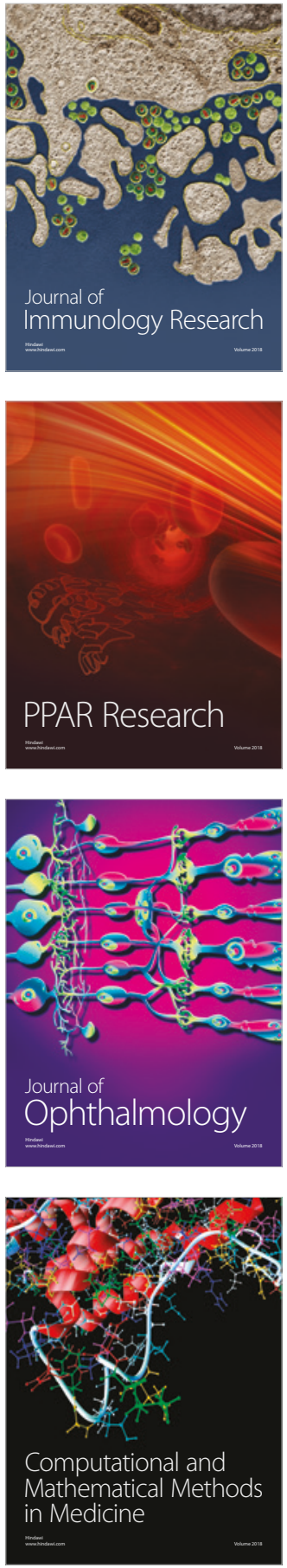

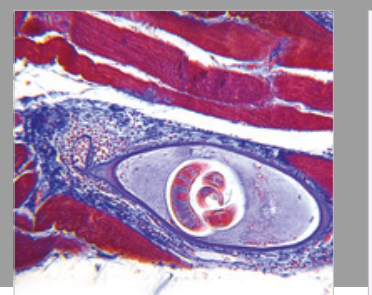

Gastroenterology Research and Practice

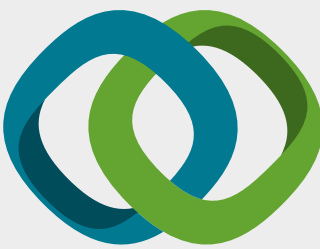

\section{Hindawi}

Submit your manuscripts at

www.hindawi.com
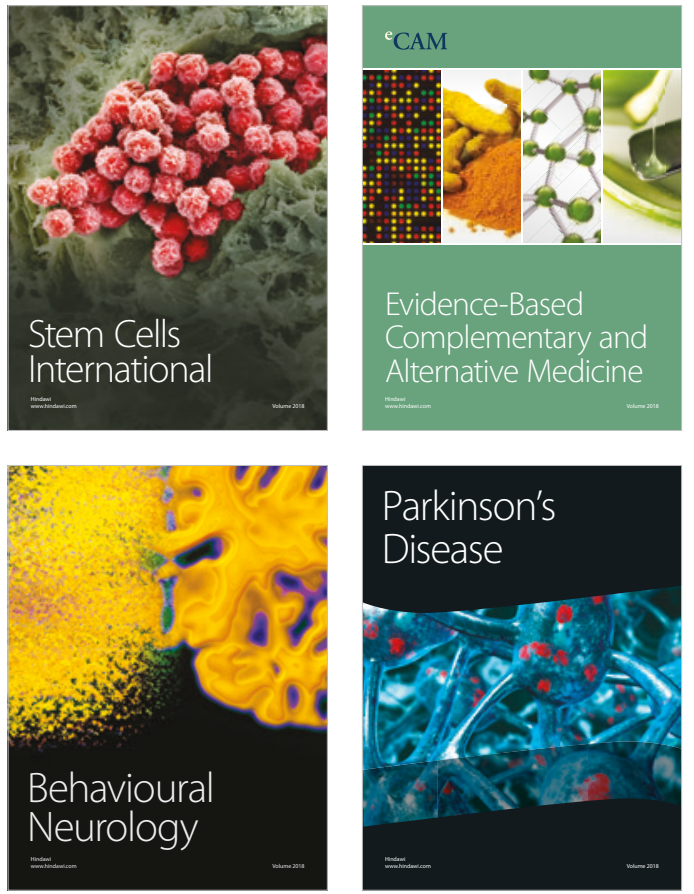

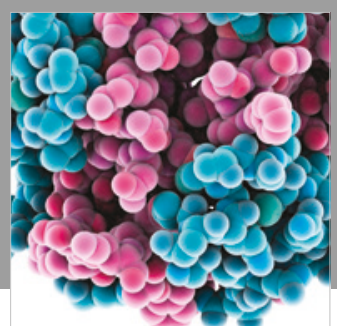

ournal of

Diabetes Research

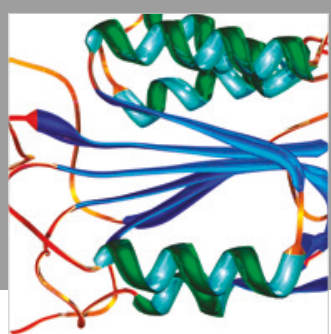

Disease Markers
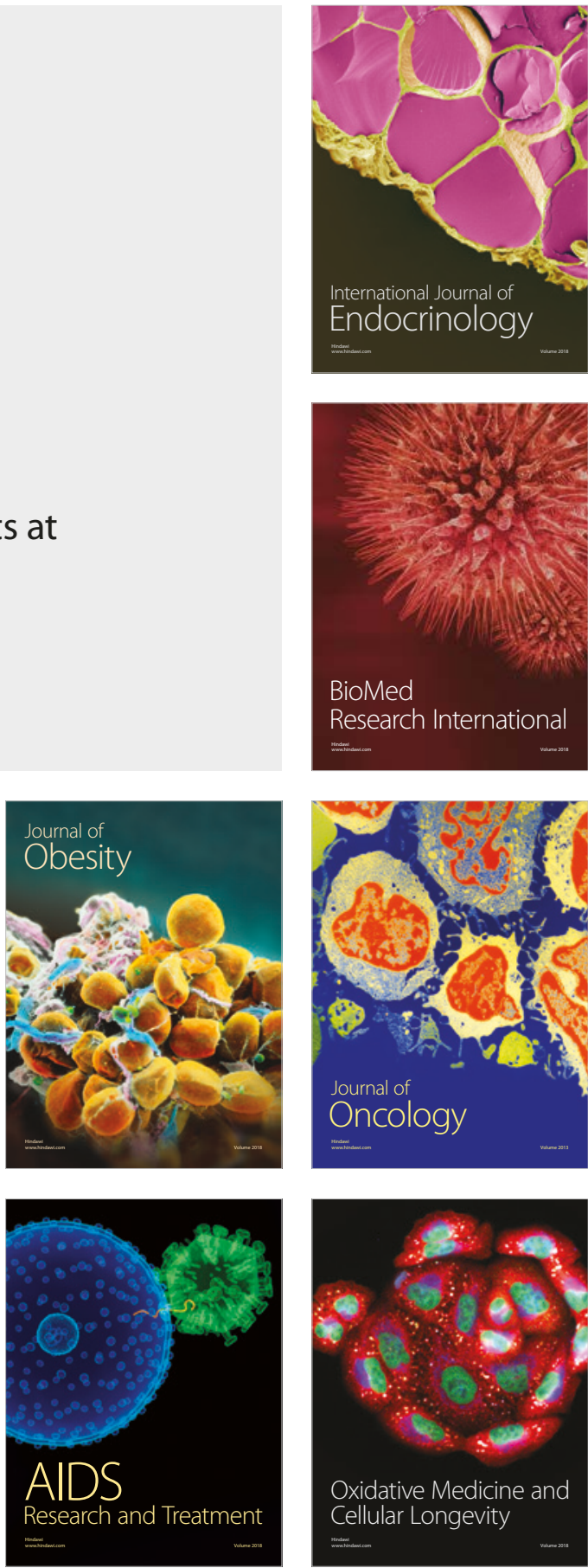